\title{
Analysis of Success Factors and Developing Potential of Pop Mart
}

\author{
Feier $\operatorname{Liu}^{1, *}, \dagger$, Linfeng Lyu $^{2, \dagger}$, Kaize Yang ${ }^{3, \dagger}$

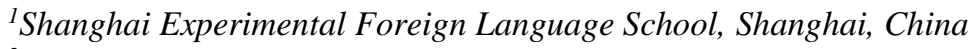 \\ ${ }^{2}$ Qingdao No.58 Senior High School, Qingdao, China \\ ${ }^{3}$ Harbin No.9 High School, Harbin, China \\ *Corresponding author. Email: guanghua.ren@gecacademy.cn \\ These authors contributed equally
}

\begin{abstract}
This study discusses the rising reason of POP MART, respectively, on the marketing strategy and IP analysis. Otherwise, it also makes a potential analysis for POP-MART to discuss the competition between substitutes and the future development of the blind box economy. For the special marketing strategy of POP MART, the Hide Styles of blind box utilize the extremely low probability and high value to seize the psychology of customers. Whatever online or offline marketing, POP MART always gains an excellent turnover. Especially in online marketing, POP MART also develops a community culture of the blind box for the amateur of a blind box. In addition, all kinds of IP combinations of Pop Mart were analyzed. Pop Mart's IP is mainly divided into designer IP and brand cooperation IP. By comparing the annual revenue generated by various IPs from 2019 to 2020, it is concluded that the popularity of blind boxes is cyclical, and Pop Mart must continue to produce IPs to maintain its dominant position in the market. The blind box makes a large revenue for the Pop Mart since these moulds are totally catered to the taste of the youth. Even more, competitors join in this market, Pop Mart is still the first choice of the consumers. However, to continuously develop the company, many projects still need to be added and improved.
\end{abstract}

Keywords: Pop Mart, Market pattern, IP, Finance

\section{INTRODUCTION}

After ten years of development, some large trendy culture entertainment companies in the Chinese trendy toy industry had matured marketization. Among them, the main outbreak point that promoted the popularity of trendy toys appears after the new type of gameplay, the blind box. China is one of the most potential consumption powers, with continuously outpace global growth rate, the blind box economy has become more and more popular in recent years. The popularity of the blind box economy directly leads to the crowded situation in the international fashion toy fairs. As one of the leading domestic blind box dealers, POP

MART has some keys to success, relatively on a number of IP and special marketing strategies. In addition, as part of the new toy industry, there is always special products value and competition between a substitute of blind box and the analysis for the future development of POP MART and blind box economy.

\subsection{The rising of POP-Mart}

Chai studied the reason for the rise of the POP MART on IP and marketing. He used the milestones in the development process to show the data from 2014. These data include the share price, the increasing market value, the net profit, the gross profit rate, and the growth rate. It can be concluded that a large number of IPs and the increasing input on marketing could be the advantages of POP MART. According to this article, the author said that the flagship's low market share gives the industry more opportunities for late delivery. Compared with the explosive growth of consumer demand, the expansion opportunity of the blind box market is still huge [1]. Wei studied the heyday and bubble of Pop-mart by doing market research and information comparison. It points out that lowering the threshold, positioning the new consumers, and dual-line operation of multi-contact were the three main reasons that could cause Pop-mart's dominance in the fashion toy market. However, at the same time, there were also some 
problems of Pop-mart, such as the lack of brand competition barriers. Wei points out that according to the market research results, consumers did not like the brand of POP-mart but the IP of Molly. Therefore, even though Pop-mart has been very successful, it still has to compete between design resources and other fields [2]. Liu did some researches about how Wang can make a pop mark a successful brand. This is because of the management. Before Wang started opening the pop mart, he set up several shops to sell all kinds of stuff. Then he began to learn more about this kind of way of selling, such as the Japanese company called "loft". The pop mart has grown fast after opening, and it started expanding abroad. Pop mart cooperated with hundreds of artists before the capitalists found it. It attracted so many consumers because of its nice management of the company. Wang knows how to catch consumers' tastes and doesn't need to be anxious [3]. Xu et al. discuss the enterprise data, operating income, operating cost, selling expenses, administrative expenses, and net profit of Pop-mart Company in China by using the method of cash flow discount model. They predicted the valuation of Pop-mart enterprise, through the discount rate method, tends to be 958 million yuan [4]. Zeng and Cheng did some researches about the comparison between the future of POP MART and the DISNEY. Wang, the founder of Pop Mart, thought that POP MART would be the next DISNEY. By comparing these two companies, the expansion of DISNEY was the historical epitome of cultural industry globalization. People Loved and approved the characters in each story, which brought American stories to global stories. However, POP MART is a brand that makes characters without stories. Wang Ning thought young people nowadays don't have so much time to accept new stories. It doesn't have stories to carry the values and philosophy. To become a company like DISNEY, POP MART still needs to capture human emotion and identify with consumers instead of having only endless creative characters [5].

\subsection{The impact of IP on the business of Pop-mart}

Su studied the huge business profits and continuous increase of POP MART. Su used a series of net profit and growth rates to show the explosive growth of POP MART. Su also analyzed that IP as one of the reasons for the explosive growth. Otherwise, Su showed her opinion on the market outlook of the blind box economy, also showed some competitive relationships related to POP MART. It shows that POP MART has a strong dependency on IP [6]. He and Tan studied and analyzed how POP MART become a big business. They used the measures and operations during the initial period of POP MART. Their conclusion shows that one of the reasons for the subsequent rise is the exploration of the niche market and shows POP MART's serious dependency on the IP [7]. Su illustrated the consumers' madness for blind box trading, which indicates that over the past year, more than 300,000 consumers have purchased blind boxes on various platforms, which caused a $320 \%$ increase in sales from the previous year at its peak. Then Su studied the competitive concept of Pop-mart with IP as the core. He finds that Pop-mart was IP-centric and had expanded into a number of niche businesses, such as co-branding with popular IPs such as Disney and Molly, which has boosted sales. Finally, $\mathrm{Su}$ gets the conclusion that the core of Pop-mart's marketing lies in IP and derivative products [8]. Zeng did some researches about the value chain of the cultural industry. Before 2015, POP MART was a shop to sell interesting things offline with low revenue. After 2015, it was affected by a toy in Japan called "Sonny Angel", it started to cooperate with artists to made creative toys and sold. It formed a close loop of the industry chain. After 2017, this company won market approval. POP MART will be a large cultural creative group about IP in the future [9].

\subsection{POP-Mart and Blind Box Economy}

Huang and Yuan studied and analyzed the reason for the rapid development of the blind box economy. Firstly, it shows the research background of the global and Chinese toy industry, respectively. They used questionnaires to understand the influence of consumers on the purchase intention of POP MART, and online, offline, and interviews were used to conduct this survey. Then they used some ways of statistics to get the data. After that, it analyzed the main reasons for the explosion of blind boxes, respectively, on the irrational consumption under the herd mentality, irrational consumption under the comparison psychology and conspicuous consumption, and the social compliance driven by IP. Then it provided some suggestions on the blind box economy than making full use of cross-field integration, updating products and designs in time, adjusting marketing strategies, giving full play to the huge commercial potential of cultural resources, and doing a good job in the protection and dissemination of cultural resources in the development [10]. Ji illustrates that the blind box economy could be so popular based on the product, consumers, and market. Among them, the psychological mechanism of consumption was particularly significant. Modern consumers, especially young individuals, were obsessed with seeking novelty. Because of the uncertainty of blind boxes, there was a huge consumer group. As John Fiske pointed out in his popular culture view, the young groups are likely to buy the same products to gain a sense of identity. The more consumers buy, the higher visibility Pop-mart could get, and the greater the economic benefits would be received by Pop-mart [11]. Chencen did a lot of researches about some questions. What is POP MART? Why is POP MART now trending in china? How about the blind box 
economy in china? Chen, the vice-dean of the Institute for Cultural Industries at Peking University, said that higher standards of living had sparked the increasing number of designer toy consumers in China. Chinese people can sell the toy-limited edition to make money. POP MART is also behind the Beijing and Shanghai Toy Show. More than 300 designers from all over the world attended the latest Beijing Toy Show [12].

According to researches about Pop Mart, to analyze its marketing, IP, the value of the products, and its development in the future. For the marketing, we will discuss the hidden style of the blind box and analyze why these series can help attract people to buy them. We will also find out how Pop Mart makes its products sell well in online marketing and offline marketing. What's more, for creating IP, IP of Pop Mart itself, designer IP, and brand corporation IP are the main part that we will focus on. This part will indicate the relationship between Pop Mart and other cooperation brands and its revenue and propriety for different IPs as well. For the last part, we focus on the value of the products, the relationship between Pop Mart and its competitors, and what are the difference between it and Disney. To become a brand like Disney, it still has so many things to do.

\section{MARKETING STRATEGY}

\subsection{The Marketing of Hiding Styles of Blind Box}

Except for the adjustment in IP, Ning Wang also added Hide Styles in the Blind Box market. The hidden version has a $0.7 \%$ chance of winning. The rarity that hides the money and high premium makes the fanatical fan of the brand get refining. Blind boxes capture the psyche of users. Surprise and excitement are caused by uncertainty. Users do not know what is inside the blind box when they buy it, and it is easy to be addicted to the instant surprise opened. The "fixed + hidden" mode adopted by merchants will further increase the excitement.

\subsection{Online Marketing}

Blind boxes have certain social attributes. Pop-mart launched a series of blind boxes, each product has its own characteristics, and it is combined into a complete series. People who buy repeated products will want to exchange with others, thus creating a social circle for users to communicate and trade. In this way, blind boxes connect people with the same interests and thus have a social attribute.

A large part of the masses in the Internet era are connected through the Internet, and they gather in a virtual community in an organized way or are active on various social platforms. The number of Blind Box players is huge, including both thousands of "advanced players" of Tibetan Babies and "Blind Box X" who just entered the pit. In the co-existence and common cyberspace, they keep a high degree of attention to the theme related to Blind Box, interact and share with each other under the same theme, and form and accumulate tens of millions or even hundreds of millions of topics. Moreover, new communication content around the theme is constantly created and becomes a large number of secondary topics, making the number of sharing groups and concern groups of blind boxes larger and the influence of blind box topics more extensive.

In addition to the IP advantage, Pop-mart discovered and promoted the community culture gradually formed due to the blind box. The brand's response was to launch its own social APP, Ba Qu. In this APP, IP image, story display, new product release, and recommendations are carried out, becoming a platform for planting grass. In addition, the APP also provides a community platform for second-hand blind box exchange and opens up a sharing area, which increases the social nature of blind boxes. Bawamat has also moved its online shopping mall to its APP, where it can place orders with one click after planting grass. Such online platforms cater to the consumption and social habits of young users. Statistics show that the APP has covered more than 600 popular brands, accounting for 49.5 percent of users and being active for more than 20 days a month. Marketing support, so that fans' stickiness and product repurchase rate greatly strengthened. Online channels are also growing rapidly. According to data, the T-mall flagship store sold over 27 million yuan worth of blind boxes on Singles' Day alone in 2018. In 2019, the sales volume of T-mall flagship store of Pop-mart reached 82.12 million yuan on the "Double 11". An increase of $295 \%$ over the same period last year ranked first in the T-mall toy category, surpassing international brands such as Lego and Bandai for the first time. In April 2020, the company announced its entry into Jingdong Supermarket. Molly ranks first among the top 10 most traded blind box products on Xianyu. In 2018, more than 230,000 Molly dolls, originally priced from 59 yuan to 79 yuan, were traded on Xianyu, with an average price of 270 yuan, with the highest price rising 39 times.

\subsection{Offline Marketing}

By the end of 2019, the company has 114 retail stores, which are the main sales channels. In 2019, the company contributed 740 million yuan in revenue while taking into account the role of building brand image and maintaining fans. Compared with retail stores, robot stores have lower upfront investment and maintenance costs and have wider coverage, reaching 825 . 


\section{IP ANALYSIS}

\subsection{IP Overview}

At the end of 2019, a company called POP-Mart, which has a valuation of 10 billion, was successfully listed in the Hong Kong stock market. Its success is inseparable from the marketing mode of its popular IP, which is mainly divided into two main parts, designer IP and brand cooperation IP. According to industry analysis, the upstream of the blind box industry chain is mainly built around IP. Wang Ning, chairman of POP-Mart, said directly that IP would be the core of their business in the next five years. According to incomplete statistics, POP- Mart has a large number of propriety IP and exclusive licensed IP. Currently, POP-Mart operates 93 IP addresses, including 12 propriety IPs, 25 exclusive licensed IPs, and 56 non-exclusive licensed IPs. And various IP products occupy over $85 \%$ of the annual income of POP-Mart in 2019.

\subsection{Designer IP}

According to the 2019 prospectus published by POP-Mart, there are three main types of IP for POPMart making profits, namely, propriety IP, exclusive licensed IP, and non-exclusive licensed IP. As can be seen from Figure 1, propriety IP accounts for $37.20 \%$ of sales, exclusive licensed IP accounts for $35.40 \%$, and non-exclusive licensed IP accounts for $9.50 \%$

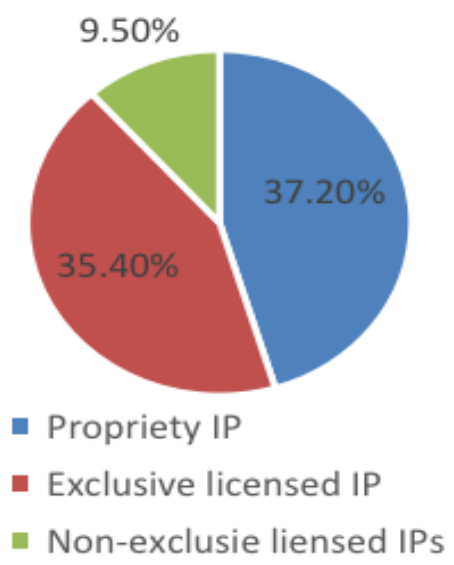

Figure 1 POP-MART brand income composition in 2019

As shown in Figure 2, Molly plays a leading role in propriety IP. Since 2016, POP-Mart has officially taken Molly's IP, mass produced it, and put it into a blind box. By 2020, Molly has generated 356.918 million yuan of income for POP-Mart, accounting for $14.2 \%$ of the total income. Among the exclusive licensed IP, pucky is the leading one. In the financial report of 2020, this exclusive licensed IP created revenue of RMB 300.009 million for POP- Mart, accounting for $11.9 \%$. Molly and pucky are not only the lifeline of POP- Mart but also a double-edged sword. As the market's pursuit of Molly and pucky IP begins to decline, POP-Mart's revenue will be greatly reduced.

Table 1. POP-MART brand income composition in 2019

\begin{tabular}{|c|c|c|}
\hline IPS & Revenue & Proportion \\
\hline $\begin{array}{l}\text { Propriety } \\
\text { products }\end{array}$ & $1,384,209$ & $82.1 \%$ \\
\hline Propriety IPs & 627,027 & $37.2 \%$ \\
\hline --Molly & 456,018 & $27.1 \%$ \\
\hline --Dimoo & 100,085 & $5.9 \%$ \\
\hline - 8080\& & & \\
\hline $\mathrm{COCO}$ & 25,454 & $1.5 \%$ \\
\hline $\begin{array}{l}\text {--Other } \\
\text { propriety IPs }\end{array}$ & 45,470 & $2.7 \%$ \\
\hline $\begin{array}{l}\text { Exclusively } \\
\text { licensed IPs }\end{array}$ & 597,362 & $35.4 \%$ \\
\hline --PUCKY & 315,318 & $18.7 \%$ \\
\hline --The Monsters & 107,846 & $6.4 \%$ \\
\hline $\begin{array}{l}\text {--SATYR } \\
\text { RORY }\end{array}$ & 63,086 & $3.7 \%$ \\
\hline $\begin{array}{l}\text {--Other } \\
\text { exclusive }\end{array}$ & & \\
\hline licensed IPs & 111,112 & $6.6 \%$ \\
\hline $\begin{array}{l}\text { Non-exclusive } \\
\text { licensed IRs }\end{array}$ & 159,820 & $9.5 \%$ \\
\hline $\begin{array}{l}\text { External } \\
\text { procurement } \\
\text { and } \\
\text { consignment }\end{array}$ & 279,986 & $16.6 \%$ \\
\hline 0thers & 19,239 & $1.3 \%$ \\
\hline Total & $1,683,434$ & $100.0 \%$ \\
\hline Table 2. POP-I & ome compos & ition in \\
\hline IPs & Revenue & Propriety \\
\hline Propriety products & $2,136,196$ & $85.0 \%$ \\
\hline Propriety IPs & 979,940 & $39.0 \%$ \\
\hline --Molly & 356,918 & $14.2 \%$ \\
\hline --Dimoo & 315,248 & $12.5 \%$ \\
\hline
\end{tabular}




\begin{tabular}{lrr}
-- BOBO\&coco & 67,415 & $2.7 \%$ \\
--SKULLPANDA & 39,065 & $1.6 \%$ \\
--Other propriety IPs & 201,294 & $8.0 \%$ \\
Exclusive licensed IPs & 711,904 & $28.3 \%$ \\
--PUCKY & 300,009 & $11.9 \%$ \\
--The Monsters & 204,828 & $8.1 \%$ \\
--SATYR.RORY & 33,684 & $1.4 \%$ \\
--Other exclusive licensed IPs & 173,383 & $6.9 \%$ \\
Non-exclusive licensed IPs & 444,352 & $17.7 \%$ \\
External procurement and & & \\
consignment & $\mathbf{3 6 8 , 4 0 0}$ & $\mathbf{1 4 . 6 \%}$ \\
Others & $\mathbf{8 , 8 7 5}$ & $\mathbf{0 . 4 \%}$ \\
Total & $2,513,471$ & $100.0 \%$ \\
\hline
\end{tabular}

By comparison with Table 1 and Table 2, from 2019 to 2020, the total turnover of Pop-mart has increased by83, 037,000 yuan. Although the turnover of its own IP has increased from RMB 627,027,000 to979, $940,000 y u a n$, the revenue gained from the main design of IP -- Molly has decreased significantly. This also shows that the popularity of blind boxes is cyclical, so Pop-mart must ensure that it can continue to produce hot style IP to continue to generate high revenue.

According to ShikeNews headlines, in 2020, POP-Mart signed more than 120 IP contracts with more diverse IP types and styles, which also means that there are still great competitions and challenges in terms of designer resources for POP-Mart. From the sales strategy of POP-Mart in recent two years, we can see that its core is to control the production source of IP, designer IP. At present, more than 30 designers have signed exclusive contracts with POP-Mart and become part of the exclusive licensed IP. However, there are still nearly 100 designers who have not signed exclusive agreements with POP-Mart. So, no matter at home or abroad, POP-Mart will still face fierce competition among excellent designer resources. Therefore, I think in the future, POP-Mart will focus on cultivating its own designers. Although doing so may lead to higher costs, it is beneficial to increase market share.

\subsection{Brand cooperation IP}

Obviously, the popularity of each IP is cyclical. In the first half of 2020, POP-Mart's main blind box, IP Molly, achieved a revenue of 357 million yuan, a decrease of $21.73 \%$ compared with the same period of the previous year. This also means that only relying on the designer IP for marketing is obviously a great risk. With the gradual sinking of the IP cash channel, POP-Mart has formed non-exclusive IP cooperation with Mickey Mouse, Marvel Universe, Despicable Me, Pet Elf, and Hello Kitty from 2019 to 2020. The cooperation with these famous international brands has also created 444.352 million yuan of income for POP-Mart in 2020.

\section{POTENTIAL ANALYSIS}

\subsection{Value of the products}

For consumers, a blind box can meet the daily emotional needs of users. When users feel upset, they can buy a blind box to relax; if they are happy, they can buy a blind box as a pastime. Blind boxes of pop mart are light and convenient, letting consumers buy online and offline. Blind box always catches up with the trend. This makes good use of the user's herd mentality. Nowadays, the largest group of the consumer is the youth. They are pursuing only material wealth but also spiritual satisfaction. POP MART corporate with many artists, and these products really catch the eyes of this group of people.

For the Pop Mart company, the cost of producing the blind box is low, and the main fixed costs are IP licensing and mold opening costs. The cost of a single mould is about 23 yuan, and it is sold at the price of 69 . The gross profit rate is around $60 \%$. So, the more a blind box is sold, the lower the cost and the higher the rate of profit. Once the blind box is purchased, consumers will repurchase because of the Gambler psychology. People always believe the one they want will come next. This makes the repurchase rate of Pop Mart reached 58\%, and the income reached 1.683 billion yuan in 2019.

\subsection{Competitions between substitutes}

In the competitive market, substitutes of the products of Pop Mart occurs. These products may have the same kinds of functions as Pop Mart but at a lower price. The demand for the Pop Mart may fall because of this. As the figure shows below, when the price of Pop Mart increase, the quantity demand for the substitutes will increase. There are many alternative brands for Pop Mart, for instance, the 52TOYS, Ruo Tai, and Sunny Angel. Pop Mart got the idea of the blind box from Sunny Angel at first, so people may have brand loyalty to it. It is hard for Pop Mart to compete.

\subsection{The future development}

If Pop Mart wants to continue expanding its business to other areas, it still needs to do many things. As a kind of collection, the product itself doesn't have real value. When time goes by, it will not appreciate like other products, such as wine. With the fast-growing of the Pop Mart, people predict that it may become the second Disney. But Disney used stories to create many 
successful characters, and these become childhood memory for kids all over the world. These stories caries values and ideas, and it attracts people to have the loyalty of this brand. To develop as Disney, Pop Mart need to have the value leading ability, emotional stickiness, and the ability to capture human emotion. This can help Pop Mart to jump out of the pure trend of commodity attributes. It can also add stories to each series of characters, making the product itself have the values of collection. To become a company like Disney, Pop Mart can rely on a single IP like Molly. It needs more "Molly" to prove itself.

Table 3. The difference between Pop Mart and Disney

\begin{tabular}{ll}
\hline Pop Mart & Disney \\
\hline $\begin{array}{l}\text { Characters don't have } \\
\text { backgrounds to attract } \\
\text { people to keep it and } \\
\text { remember it. }\end{array}$ & $\begin{array}{l}\text { Have stories to support each } \\
\text { character, and people can always } \\
\text { remember them. }\end{array}$ \\
$\begin{array}{l}\text { Over reliance on a single } \\
\text { IP, the proportion of } \\
\text { income earned by Molly } \\
\text { and Pucky reached about }\end{array}$ & $\begin{array}{l}\text { always create new IP, which let } \\
\text { 50\% }\end{array}$ \\
$\begin{array}{l}\text { Lack of "personalized" } \\
\text { IP, lack of IP operation } \\
\text { experience, products are } \\
\text { mainly fashioned toys, } \\
\text { relatively single }\end{array}$ & $\begin{array}{l}\text { Formed a relatively complete } \\
\text { industrial chain, Media network, } \\
\text { theme park, film, and related } \\
\text { products and interactive business } \\
\text { sectors are interrelated and } \\
\text { promoted. }\end{array}$ \\
\hline
\end{tabular}

\section{CONCLUSION}

In conclusion, we discuss the marketing strategy of Pop Mart, such as online and offline marketing, and the reason why hide style is so popular that it can always attract people to consume. Also, studying all kinds of Pop Mart's IP combinations and comparing the revenue earned by Pop Mart between 2019 and 2020. This part indicates that the connection between Pop Mart and other corporative brands. What's more, the value of the products, the relationship between competitors, and future development is an area that we focus on. If Pop Mart wants to expand its business in the future, it still needs to find the opportunity to improve the way of marketing and the personification of the products. It also needs to export its IPs to show its work to the world and strive for more innovative partners.

\section{REFERENCES}

[1] Chai Qiaoshan.(2021). From the grocery store to the blind box faucet.Brand China (03),68-70. doi:CNKI:SUN: zgpp.0.2021-03-020.
[2] Wei Qiao.(2021). POP-Mart's heyday and bubble.China Marketing(02),32-34. doi:CNKI:SUN:XSYS.0.2021-02-011.

[3] Liu Yuanlong.(2018).Pop mart's trendy toy Empire.fairly well-off(24),60-62. doi:CNKI:SUN:CHXK.0.2018-24-029.

[4] Xu Jing, Wang Yinuo, Tang Yunqing, Wang Hening.(2021).Analysis of Enterprise Value Based on Free Cash Flow Discount Model -- Taking Beijing POP- Mart as an example. Trade Fair Economy(06),106-108. doi:CNKI:SUN:SZJJ.0.2021-06-034.

[5] Zeng Xin \& Chen Xian.(2020).“Chinese Disney"' sIP's way of "going abroad": Brand marketing of art toy, competitive barriers and reflection on internationalization . International brand observation (34),65-67. doi:CNKI:SUN:GJPC.0.2020-34-023.

[6] Su Qi.(2020). The secret of tide playing the first share, Popmart's profiteering business.Modern Commercial Bank (14),76-79. doi:CNKI:SUN: jrxk.0.2020-14-021.

[7] JiPai He.(2020).POP MART "Breaking a Circle". The 21st Century Business Review(07),66-69. doi:CNKI:SUN:ESYS.0.2020-07-016.

[8] Su Muhui.(2019. Blind box meets IP consumption: two-way empowerment or $\$ 100$ billion market explosion.New Industrial Economy(10),66-69. doi:CNKI:SUN:XCHJ.0.2019-10-022.

[9] ZengWangsheng.(2018).Analysis of cultural and creative projects from the perspective of cultural industry value chain--Take pop Mart as an example. Modernization of the market.(10),11-12. doi:10.14013/j.cnki.scxdh.2018.10.005.

[10] Huang, F.M., \& Yuan, J.C.(2021). Analysis of the reasons for the rapid development of blind box industry: A case study of "Bublimat".Circulation of the national economy (03), 21-23, doi: 10.16834 / j.carol carroll nki issn1009-5292.2021.03.007.

[11] JI Ruyi.(2020).psychological analysis of "blind box economies" consumption from the perspective of Internet communication.Southeast Communication(01),117-119. doi:10.13556/j.cnki.dncb.cn35-1274/j.2020.01.037.

[12] Chencen https://daxueconsulting.com/pop-mart-designer-toy -market-in-china 